5-22-2009

\title{
Mechanics-Based Model for Non-Affine Swelling in Perfluorosulfonic Acid (PFSA) Membranes
}

\author{
Ahmet Kusoglu \\ University of Delaware \\ Michael H. Santare \\ University of Delaware, santare@udel.edu \\ Anette M. Karlsson \\ Cleveland State University, a.karlsson@csuohio.edu
}

Follow this and additional works at: https://engagedscholarship.csuohio.edu/enme_facpub

Part of the Mechanical Engineering Commons, and the Membrane Science Commons How does access to this work benefit you? Let us know!

\section{Publisher's Statement}

NOTICE: this is the author's version of a work that was accepted for publication in Polymer.

Changes resulting from the publishing process, such as peer review, editing, corrections, structural formatting, and other quality control mechanisms may not be reflected in this document. Changes may have been made to this work since it was submitted for publication. A definitive version was subsequently published in Polymer, 50, 11, (05-22-2009); 10.1016/ j.polymer.2009.03.045

\section{Original Citation}

Kusoglu, A., Santare, M. H., and Karlsson, A. M., 2009, "Mechanics-Based Model for Non-Affine Swelling in Perfluorosulfonic Acid (PFSA) Membranes," Polymer, 50(11) pp. 2481-2491.

This Article is brought to you for free and open access by the Mechanical Engineering Department at EngagedScholarship@CSU. It has been accepted for inclusion in Mechanical Engineering Faculty Publications by an authorized administrator of EngagedScholarship@CSU. For more information, please contact library.es@csuohio.edu. 


\title{
Mechanics-based model for non-affine swelling in perfluorosulfonic acid (PFSA) membranes
}

\author{
Ahmet Kusoglu, Michael H. Santare*, Anette M. Karlsson \\ Department of Mechanical Engineering, University of Delaware, 126 Spencer Laboratory, Newark, DE 19716, United States
}

\section{Introduction}

Ionomers are ion-containing polymers that exhibit unique conductivity, permeability and electro-chemical properties. Ionomers are of great practical interest because of their extensive use in electro-chemical devices [1-3]. Perfluorinated sulfonic acid (PFSA) ionomers are of particular interest, since they are commonly used as ion-conducting electrolytes in proton exchange membrane fuel cells due to their inherent electro-chemical properties and mechanical integrity $[1,4,5]$. Chemically, PFSA ionomer is composed of a polytetrafluoroethylene (PTFE)-like backbone and (perfluorovinyl ether) side-chains terminated with sulfonic acid endgroups $\left(\mathrm{SO}_{3}^{-}\right)$, where exchangeable ions can attach. During fuel cell operation, water is formed as a by-product. In the presence of water, PFSA membranes swell, resulting in enhanced ion (e.g. proton) conductivity, which is necessary to sustain desirable cell performance [6-13]. However, in addition to adequate water management, reliable, long-term operation requires mechanical

\footnotetext{
* Corresponding author. Tel.: +1 3028312246.
}

E-mail address: santare@udel.edu (M.H. Santare). stability for the membranes and swelling also plays a key role in the mechanical response of the fuel cell membranes [1,14-16]. Thus, understanding the sorption behavior of PFSA ionomer membranes and its effects on the mechanical properties remains a key issue to the development and characterization of PEMFCs.

A number of studies of the morphology of swollen-PFSA membranes suggest a phase-separated nanostructure where the water molecules reside in hydrophilic domains, the so-called clusters, embedded in a polymer matrix with sulfonic acid $\left(\mathrm{SO}_{3}^{-}\right)$endgroups located along the interface $[11,17-25]$. In a dry PFSA membrane, ionic $\mathrm{SO}_{3}^{-}$groups still cluster to minimize the free energy $[18,26,27]$. This type of two-phase nanostructure was also examined and verified in a number of theoretical studies [7,28-31]. However, the geometrical interpretation of the nanostructure is still a subject of debate (for a discussion of the nanostructure see Refs. $[5,17,28])$. For example, in addition to the spherical cluster network model proposed earlier by Gierke, Hsu and co-workers [19,32], there are other studies describing the nanostructure of highly swollen PFSA as comprised of rod-like polymeric aggregates with water pools residing among them $[11,33]$ and, recently, as parallel cylindrical water-filled nano-channels surrounded by polymer backbone [28]. 
In any case, the swelling of PFSA membranes is commonly described as a microscopic process in which water molecules attach to the hydrophilic sulfonic acid $\left(\mathrm{SO}_{3}^{-}\right)$end-groups to form water-filled domains. These water-filled hydrophilic domains are separated from the hydrophobic polymer matrix resulting in a two-phase nanostructure as observed through a vast number of scattering experiments [9,20-25,34-37]. Upon water uptake, water molecules initially ionize the $\mathrm{SO}_{3}^{-}$groups and remain bound to them. Additional water molecules are then free to move through the ionomer, causing growth of the clusters and consequently the macroscopic swelling of the membrane $[8,9,11,24,34,38-41]$.

As the clusters grow, their volume and surface area increase leading to deformation of the surrounding polymer matrix. The polymer matrix, in turn, exerts a counter force due to its elastic deformation, thereby limiting the swelling [7,11,18,26,27,40-42]. Thus, the swollen state represents an equilibrium between two opposing forces: (i) the osmotic swelling pressure driving the water volume to increase, and (ii) the elastic deformation of the polymer matrix which exerts a counter pressure on the water-filled hydrophilic domains driving water volume to decrease $[11,18,20,30,40,41,43]$. As a result of this interplay between water uptake and matrix deformation during swelling, a continuous structural reorganization and coalescence of the water-filled domains occur at the nanoscale. Consequently, the magnitude of the strain of the individual water-filled nano-domains is higher than that of the overall macroscopic swelling strain of the material. This relationship is referred to as non-affine deformation and has been shown to occur for PFSA membranes by a number of studies in literature [11,18-22,29,33-35]. Despite the growing body of literature on the sorption behavior $[7,8,10,24,34,35,38,40,44-50]$ and nano-structural modeling and characterization [11,13,17$22,28,29,31,33-36,39,49,51,52]$ of PFSA ionomers, the relationship between the water uptake and nanostructure, temperature, mechanical properties and membrane pretreatment is not well established. Understanding of these issues requires a general, yet fundamental model, which is the subject of this study. The theoretical models available in the literature for PFSA use either mechanical models (with spheres representing the water domains) that assume affine swelling $[18,41,53]$, or statistical approaches that assume non-affine swelling [54]. In the current study, we will develop a geometry-dependent mechanical modeling framework, which starts with non-affine swelling and that can be used to determine the relationship between the swelling pressure and the water uptake, which is crucial to the characterization of the sorption behavior of (PFSA) ionomers.

\section{Previous models in literature}

The theoretical models currently available in the literature for describing the equilibrium swelling in polymers are largely based on the statistical theory of rubber elasticity. One of the earliest models, developed by Flory [55], assumes that the entropy gain, due to the mixing of the polymer and solvent, is equal to the loss of conformational entropy due to the stretching out of the polymer chains. Following this assumption, the change in the free energy due to the elastic deformation of the swollen polymer, $\Delta F_{\mathrm{el}}$, can be calculated for a given representation of the polymer chain length distribution. The swelling pressure is then, $P=\leftrightarrow\left(\Delta F_{\mathrm{el}}\right) / \partial\left(1 / \phi_{\mathrm{p}}\right) \leftarrow$ with $1 / \phi_{\mathrm{p}}=V / V_{0}$ being the reciprocal of the polymer volume fraction, i.e. the ratio of the swollen volume to the initial dry volume [55]. For example, for an isotropically swollen Gaussian polymer network, with end-to-end polymer chain distance represented by a Gaussian distribution, swelling pressure is [55]
$P=\leftarrow\left(\phi_{\mathrm{p}}^{1 / 3}-\frac{1}{2} c \phi_{\mathrm{p}}\right)$

where $G$ is the shear modulus of the polymer network. The parameter $c$ can be chosen to be either the "affine limit" $(c=1)$, where there is an additional gain in the entropy due to the volume change, or the "phantom limit" $(c=0)$, representing a hypothetical network whose chains and junctions may move freely through one to another. Analogous expressions for the free energy have also been proposed for non-Gaussian network representations for swollen polymers $[43,56]$.

While there are a number of studies which relate the swelling pressure increase with increasing water volume fraction, the specific form of this relationship is different in different models [43,53,56-61]. Most of these models are based on the assumption of affine swelling, which means that there is no structural reorganization at the molecular scale and that the junction points between polymer chains move proportionally to the macroscopic swelling deformation. However, as mentioned in Introduction, it has been observed that PFSA ionomer membranes exhibit nonaffine swelling, i.e. there is structural reorganization and the strain of the micro-domains is not the same as that of the macroscopic strain. These affine models do not describe the non-affine swelling behavior observed in PFSA membranes.

Dreyfus [27] developed a model for the adsorption of water around an electrical charge surrounded by deformable polymer matrix. Also, in their work on sorption in PFSA, Hsu and Gierke [18] and Mauritz and Rogers [41], assumed the equilibrium swelling pressure to be equal to the pressure required to increase the radius of a spherical cavity embedded in an infinite elastic medium whose modulus is equal to that of the polymer (i.e. PFSA membrane). However, this simplification still does not take into consideration the structural reorganization of the polymer chains and water clusters during swelling, as discussed elsewhere [54]. Thus, the classical elasticity approach cannot be used to determine the swelling pressure in structures that exhibit a non-affine swelling behavior.

A study that takes into account the non-affine swelling of PFSA, was conducted by Freger [54] who developed an expression for the free energy of a two-phase swollen-polymer by replacing the assumed affine deformation of each chain in the Flory-Rehner model [55] with the affine deformation of unit cells, which inflate upon water uptake. In this model, the swollen matrix is represented by polyhedral cells generated by Voronoi tessellation where each cell contains one hydrophilic water-filled domain. During swelling, the faces of each cell are assumed to undergo a shape-preserving uniaxial compression such that the stretch ratio in the in-plane directions is equal to the macroscopic dimensional change, $\Lambda_{\text {in-plane }}=\phi_{\mathrm{p}}^{-1 / 3}$, while the cells are compressed in the thickness direction such that the cell volume remains constant. This set of constraints results in an overall non-affine deformation for the membrane. From this set of assumptions, the swelling pressure can be expressed as [54]

$P=\frac{2}{3} G\left(\phi_{\mathrm{p}}^{1 / 3}-\phi_{\mathrm{p}}^{7 / 3}\right)$

This model predicts vanishing pressure for a dry membrane and a linear increase in the swelling pressure with volume change at low water content as observed in previous studies [59,61].

In this paper, we will develop an alternative mechanics-based model for the swelling pressure which begins with non-affine deformation to characterize the effects of the structural reorganization due to the coalescence of the water clusters. First, we will discuss the two representative volume elements (RVEs) we use to characterize the two-phase nanostructure of a swollen-PFSA 
membrane. The RVEs are based on the body of nano-structural descriptions given in the literature. Then, a non-affine swelling mechanical model is proposed for capturing the deformation of PFSA membrane during swelling. From this, an expression for the swelling pressure is developed as a function of the water volume fraction, temperature and Young's modulus of the dry membrane. In order to further investigate the model parameters and material properties used in the model, the proposed formulation is used to obtain theoretical sorption isotherms for predried and non-predried membranes and a comparison of these, with data from the literature is presented.

\section{Proposed model}

\subsection{Assumptions}

Our proposed model is based on the following assumptions:

(i) The nanostructure of PFSA membrane is homogenized and represented by equal-sized RVEs of spherical or cylindrical shape, with an inner domain of water surrounded by a concentric, outer domain of polymer. The ratio of inner to outer radius of the RVE can vary, and is determined by the water volume fraction.

(ii) Non-affine swelling of the membrane is used as input to the model. Consequently, the outer radius of the RVE is determined based on the relationship between the inter-cluster distance and the macroscopic water volume fraction (which will be described in detail in Section 4). The non-affine swelling behavior is assumed to be independent of temperature.

(iii) Non-affine deformation is here associated with the structural reorganization at the nanoscale [11,18-21,34,35]. Alternatively, this type of deformation could also be attributed to the lamellar dilution of rod-like particles [33] or to randomness in the nanostructure at different scales.

(iv) Swelling is assumed to be a quasi-static process such that the membrane is in equilibrium at each given water volume fraction. The instantaneous size of the RVE is determined accordingly, by considering the coalescence of the clusters. However, we do not attempt to calculate how the equilibrium is reached or how the structural reorganization occurs. Thus, molecular level electro-chemical interactions among the water molecules and, ionic groups and polymer matrix are not considered here.

(v) The deformation behavior of the polymer matrix, due to the growth of the clusters, is modeled by idealized elastic mechanical springs without explicit consideration of the molecular structure of the polymer (e.g. thermodynamical treatment of the polymer chains).

\subsection{Representative volume element}

As mentioned earlier, several models exist in the literature for the nanostructure of PFSA membranes. There appears to be a lack consensus among researchers [5,17,28], so we will study two commonly used models (i) spherical cluster model, and (ii) cylindrical channel model. Therefore, we will also be able to investigate the effect of the model geometry on the swelling behavior.

\subsubsection{Spherical cluster model}

One of the earliest models for the nanostructure of PFSA ionomers is the cluster-network model proposed by Gierke and Hsu $[9,18,19]$. They used a simplified geometric representation: a polymer matrix containing a cubic array of spherical, inverted micellar clusters which grow upon water uptake [19]. This growth corresponds to an increase in the volume of the cubic lattice, causing an increase in the number of $\mathrm{SO}_{3}^{-}$groups per cluster due to the cluster coalescence (i.e. non-affine swelling).

In this study, the membrane volume is assumed to be nearly filled by identical, uniformly distributed spherical RVES (S-RVES), with the small remainder of the volume assumed to have identical properties to the bulk material (Fig. 1A). This structure is similar to the composite sphere assemblage concept of Hashin [62] who, using this approach, showed that the volume averaged stressstrain relation for the isotropic elastic body also represents the deformation of each of the composite spheres. For PFSA membranes, this assumption implies that the clusters have some local order as suggested by Dreyfus et al. [22]. Each spherical RVE has an (outer) radius $b$ and contains a spherical cluster of (inner) radius $a$, where the water molecules and the sulfonic acid groups reside (Fig. 1B). The inner and outer radii of the RVE can change, but due to the geometrical assumptions, their ratio is always equal to the cube-root of the volume fraction of the cluster, $\phi_{c}$,

$$
\bar{b}^{n}=\phi_{\mathrm{c}}=\phi_{\mathrm{w}}+\phi_{\mathrm{SO}_{3}} \text {, }
$$

where $\phi_{\mathrm{w}}$ and $\phi_{\mathrm{SO}_{3}}$ are the volume fractions of water and sulfonic acid groups, respectively, and $n=3$ is the geometric factor.

In our previous work [63] we developed a nano-mechanics model to describe the variation of Young's modulus for swollenPFSA membranes. In that study, we found that the inclusion of small cylindrical channels connecting the spheres is the best representation of the nanostructure for predicting Young's

\section{A Composite Sphere Assemblage Approach}

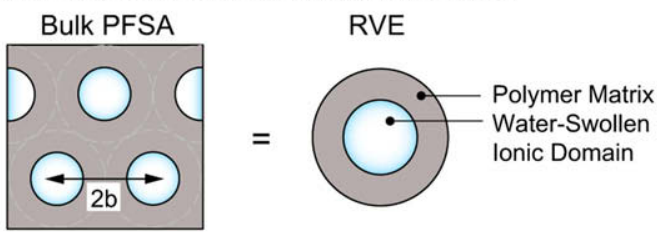

B Spherical RVE (shown with springs)

C Cylindrical RVE
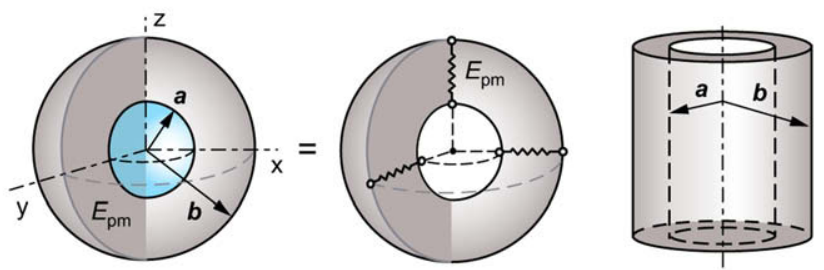

\section{Characterization of Deformation of Springs (Polymer Matrix)}

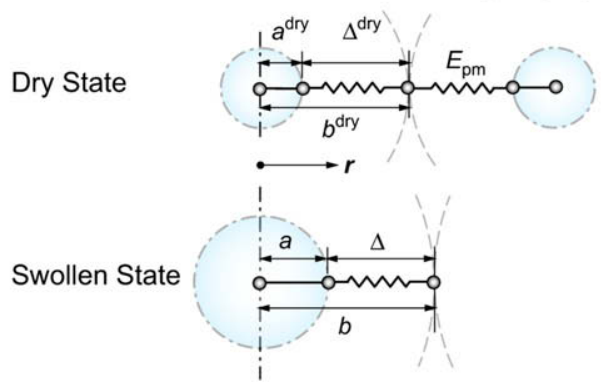

Fig. 1. (A) A representative sketch for the composite sphere assemblage approach [62] and the representative volume elements (RVEs) for the nanostructure of the swollenPFSA membrane: (B) Spherical RVE (S-RVE) and (C) the cylindrical channel, respectively. (D) Detailed sketch for the position of the elastic spring (on any orientation) with corresponding cluster and RVE radii given in dry and swollen states. 
modulus in humid air. However, in this study, we will use this simplified spherical RVE without channels to model the nanostructure of PFSA membranes for the purpose of calculating the swelling pressure.

\subsubsection{Cylindrical channel model}

In a recent study, Schmidt-Rohr and Chen [28], numerically simulated previously published scattering data of water-swollen PFSA membranes to evaluate and compare the existing morphological models. They concluded that the nanostructure of highly swollen-PFSA membranes can be best described by parallel, cylindrical water channels randomly distributed in a polymer backbone with the hydrophilic $\mathrm{SO}_{3}^{-}$ions located along the water-polymer interface. During water sorption, they concluded that the channel diameters increase and coalesce with neighboring channels. They reported that the water channel diameters in the water-swollen membrane are between 1.8 and $3.5 \mathrm{~nm}$, with an average of $2.4 \mathrm{~nm}$. Based on this nanostructure, we propose a second, cylindrical RVE of length $h$ that contains an inner cylinder occupied by water and hydrophilic end-groups surrounded by the hydrophobic polymer matrix backbone (Fig. 1C). Following an approach similar to that of spherical RVEs, the final nanostructure of PFSA membrane can be represented by identical, uniformly distributed cylindrical RVEs (C$R V E S$ ) with the small remaining volume assumed to have identical properties to the bulk material. For this geometry, $n=2$ in Eq. (3). Note that, for a dry membrane, Eq. (3) gives the inner and outer radii in dry state, $a^{\text {dry }}$ and $b^{\text {dry }}$, respectively, for the cluster volume fraction $\phi_{\mathrm{c}}^{\mathrm{dry}}$.

\subsection{Characterization of non-affine swelling}

The size and the shape of the hydrated ionic aggregates in PFSA membranes are generally investigated through (X-ray or neutron) scattering experiments. It is well established that the swelling of PFSA membranes is non-affine. For example, the microscopic swelling strain (defined as the inter-cluster distance) is much larger than the macroscopic swelling strain (which is related to the water content of the membrane) [11,18-21,29,33-35]. In this study, since we assume that the volume is nearly filled by close-packed RVEs of the same size, the inter-cluster distance is the outer diameter of the RVEs, $2 b$. Thus, upon water uptake, the dry radius of the spherical $\mathrm{RVE}, b^{\text {dry }}$, increases to the swollen radius for a given polymer volume fraction, $b=b\left(\phi_{\mathrm{p}}\right)$, and therefore the microscopic swelling strain can be defined as:

$\varepsilon^{\mathrm{s}}=\frac{b}{b^{\mathrm{dry}}}-1$.

We can also define the macroscopic swelling strain, $\Sigma^{\mathrm{s}}$, as:

$\Sigma^{\mathrm{S}}=\phi_{\mathrm{p}}^{-1 / 3}-1={\frac{V}{V_{\mathrm{p}}}}^{1 / 3}-1$

where $V$ and $V_{\mathrm{p}}$ are the swollen and initial (dry) volumes of the polymer, respectively. Consequently, $\phi_{\mathrm{p}}$ is the polymer volume fraction of the swollen membrane. Now, let the relationship between macroscopic and microscopic swelling strains be

$\varepsilon^{\mathrm{S}}=\kappa \Sigma^{\mathrm{S}}=\kappa \phi_{\mathrm{p}}^{-1 / 3}-1$,

where $\kappa$ is the non-affine swelling ratio. We can use Eqs. (4)-(6) to describe the cylindrical RVE model as well, by assuming that the swelling strain in the thickness direction is equal to the strain in inplane directions, i.e. $b / b^{\text {dry }}=h / h^{\text {dry }}$ with $h^{\text {dry }}$ being the length of the cylindrical channel RVE in the dry state.
When the membrane is completely dry (i.e. no water resides in the membrane initially), we assume that the clusters contain only the $\mathrm{SO}_{3}^{-}$groups. Therefore, $\phi_{\mathrm{c}}^{\text {dry }}$ must be equal to the $\mathrm{SO}_{3}^{-}$volume fraction of a dry PFSA membrane, i.e.

$\phi_{\mathrm{c}}^{\mathrm{dry}}=\frac{\bar{V}_{\mathrm{SO}_{3}}}{\bar{V}_{\mathrm{p}}}=\frac{\bar{V}_{\mathrm{SO}_{3}}}{\mathrm{EW} / \rho_{\mathrm{p}}}$,

where $\bar{V}_{\mathrm{SO}_{3}}$ and $\bar{V}_{\mathrm{p}}$ are the molar volumes of the ionic $\mathrm{SO}_{3}^{-}$groups and the polymer, respectively, $\rho_{\mathrm{p}}$ is the density of the dry polymer, and $\mathrm{EW}$ is the equivalent weight of the membrane, given in grams of dry polymer per mole of $\mathrm{SO}_{3}^{-}$group. Furthermore, since the terms on the right hand side of Eq. (7) are known for a given ionomer ( $\bar{V}_{\mathrm{SO}_{3}}$ can be taken to be $40.94 \mathrm{~cm}^{3} / \mathrm{mol}$ [19]), the cluster volume fraction can be determined directly from the water volume fraction, $\phi_{\mathrm{w}}$

$\phi_{\mathrm{c}}=\phi_{\mathrm{w}}+\left(1-\phi_{\mathrm{w}}\right) \phi_{\mathrm{c}}^{\mathrm{dry}}$.

Because of the structural reorganization associated with the nonaffine deformation, the volume of the polymer in the RVE must increase during water uptake, such that the (microscopic) polymer volume fraction of the RVE is always equal to the membrane's overall (macroscopic) polymer volume fraction. This assumption automatically takes into account the change in the number of sulfonic acid groups per RVE due to coalescence of clusters. Since the number of $\mathrm{SO}_{3}^{-}$groups per cluster (or RVE), $\mathrm{N}_{\mathrm{SO}_{3}}$, is always related to the polymer volume within that RVE, the increase in $\mathrm{N}_{\mathrm{SO}_{3}}$ from the dry state to any swollen state can be calculated from the volume change of the RVE and its polymer volume fraction, i.e.

$\frac{N_{\mathrm{SO}_{3}}}{N_{\mathrm{SO}_{3}}^{\text {dry }}}=\frac{\phi_{\mathrm{p}}}{\phi_{\mathrm{p}}^{\text {dry }}} \frac{V_{\mathrm{RVE}}}{V_{\mathrm{RVE}}^{\text {dry }}}=\frac{\phi_{\mathrm{p}}}{\phi_{\mathrm{p}}^{\text {dry }}}{\frac{b}{b^{\text {dry }}}}^{3}=\frac{\phi_{\mathrm{p}}}{1}\left[f\left(\phi_{\mathrm{p}}^{-1 / 3}-1\right)+1\right]^{3}$.

where $\phi_{\mathrm{p}}^{\mathrm{dry}}=1$ in a dry polymer. When $\kappa=1$ in Eq. (6), the microscopic and the macroscopic swelling both are equal to $\phi_{\mathrm{p}}^{-1 / 3}-1$, which represents affine swelling. In this case, the clusters do not coalesce $\left(N_{\mathrm{SO}_{3}}=N_{\mathrm{SO}_{3}}^{\text {dry }}\right)$. However, if $\kappa>1$, then the number of $\mathrm{SO}_{3}^{-}$ groups per cluster increases during water uptake $\left(N_{\mathrm{SO}_{3}}>N_{\mathrm{SO}_{3}}^{\mathrm{dry}}\right)$ due to the coalescence of the clusters (i.e. structural reorganization). As a result, both the total volume and the total number of $\mathrm{SO}_{3}^{-}$groups in the RVE increase during swelling, which is in agreement with the observations from various experiments [11,18,19,21,22,35] and the degree of cluster coalescence is therefore attributed to the nonaffine swelling ratio $\kappa$. The derivation of Eq. (9) is purely geometrical, and based on the observed phenomenon of non-affine swelling deformation during sorption. The energy associated with coalescence is not taken into account. During desorption of a saturated membrane, the compressive pressure in the polymer matrix will decrease, and the clusters will de-coalesce. Given a detailed description of this behavior, the model could equally well predict pressures during desorption. An in-depth investigation on the modeling of sorption-desorption cycles is left for future research.

\subsection{Proposed model for swelling pressure}

We now propose a method to calculate the swelling pressure as a function of water (or polymer) volume fraction, using a discreteparameter mechanics approach, based on the nanostructure described earlier. In order to characterize the deformation of the polymer matrix due to the growth of the clusters, the polymer matrix within the RVE is idealized mechanically, as linear spring elements. The spring constant is related to Young's modulus of the polymer matrix, $E_{\mathrm{pm}}$, thus allowing us to calculate a force per unit 
area per unit length change for the deformation. Each linear spring is placed between virtual nodes located at the surface of the cluster (at $r=a$ ) and the outer surface of the RVE (at $r=b$ ). Here, the node is shared by another spring that belongs to the neighboring RVE (Fig. 1B,D). Thus, the polymer matrix is comprised of nodes that can move in a non-affine manner during swelling and are connected by linear spring elements. In general, the PTFE-based polymer matrix is comprised of polymer chains and crystallites. However, the exact nature of the polymer matrix is irrelevant to our calculations, since we model it as elastic springs. This is analogous in some ways to the classical characterization of the deformation of polymer chains in elastically deformed polymers [64-66].

For a dry membrane, we assume that each spring is initially stress-free and has a length of $\Delta^{\text {dry }}=b^{\text {dry }}-a^{\text {dry }}$. When the membrane is exposed to an external water (vapor/liquid) source, water uptake begins and the radii of the cluster and the RVE increase to $a$ and $b$, respectively. Thus, the deformed length of the spring upon water uptake is $\Delta=b-a$ (Fig. 1D). Then, with the help of Eq. (3), the stretch ratio in the spring due to the swelling, $\Lambda_{\mathrm{pm}}$, can be written as

$\Lambda_{\mathrm{pm}}=\frac{\Delta}{\Delta^{\mathrm{dry}}}=\frac{b-a}{b^{\mathrm{dry}}-a^{\mathrm{dry}}}=\stackrel{b}{b^{\mathrm{dry}}}\left[\frac{1-\phi_{\mathrm{c}}^{1 / n}}{\left(-\phi_{\mathrm{c}}^{\mathrm{dry}}\right.}\right]$.

where $\phi_{c}^{\text {dry }}$ is the cluster volume fraction in the dry membrane. Alternatively, the "dry" value can be replaced by any chosen initial value to determine the relative stretch between any two states of hydration. A compressive force is generated in the spring (polymer matrix) due to the compressive radial strain of the material between the clusters. The change in the compressive force per unit area, can be defined as the swelling pressure, $\Delta P$, in the RVE (defined positive)

$\Delta P=P-P_{0}=-E_{\mathrm{pm}}\left(\Lambda_{\mathrm{pm}}-1\right)$

where $\Lambda_{\mathrm{pm}}-1$ is the strain in the deformed polymer matrix and $P_{0}$ is the value of the pressure in a dry membrane which will be a function of the thermal and mechanical histories. Therefore, we will examine the change in the pressure, $\Delta P$, which is related to the change in the water content upon sorption.

Using Eqs. (4), (6) and (10), the change in swelling pressure in Eq. (11) can be rewritten as

$\left.\left.\frac{\Delta P}{E_{\mathrm{pm}}}=\leftarrow 1+\kappa \phi_{\mathrm{p}}^{-1 / 3}-1\right)\right]\left(\frac{1-\phi_{\mathrm{c}}^{1 / n}}{1-\phi_{\mathrm{c}}^{\mathrm{dry}^{1 / n}}}\right)(-(1$.

It follows from Eq. (12) that the swelling pressure is only a function of the water (or cluster) volume fraction for a given set of structural properties, i.e. non-affine swelling ratio and RVE (spherical or cylindrical water-filled domains).

In the above derivation, $E_{\mathrm{pm}}$ is interpreted as Young's modulus of polymer matrix alone, since within the framework of this study, the tendency of the cluster to grow is counter-balanced by the pressure applied by the polymer matrix only (excluding the clustered ionic groups). Therefore, $E_{\mathrm{pm}}$ is the modulus of a hypothetical material; the polymer backbone of the PFSA membrane (backbone and sidechains), whose properties cannot be measured directly. However, we can estimate the relationship between $E_{\mathrm{pm}}$ and Young's modulus of dry membrane (backbone, side-chains and ionic groups), $E_{\mathrm{dry}}$, which is a measurable bulk property. This relationship can be estimated using the micro-mechanics approach in our previous work, from the cluster volume fraction and the geometry of the RVE in the dry state, and by assuming that the cluster (consisting solely of $\mathrm{SO}_{3}^{-}$ groups) has zero stiffness relative to the polymer backbone [63]. For the RVEs used in this study, this relationship can be written in

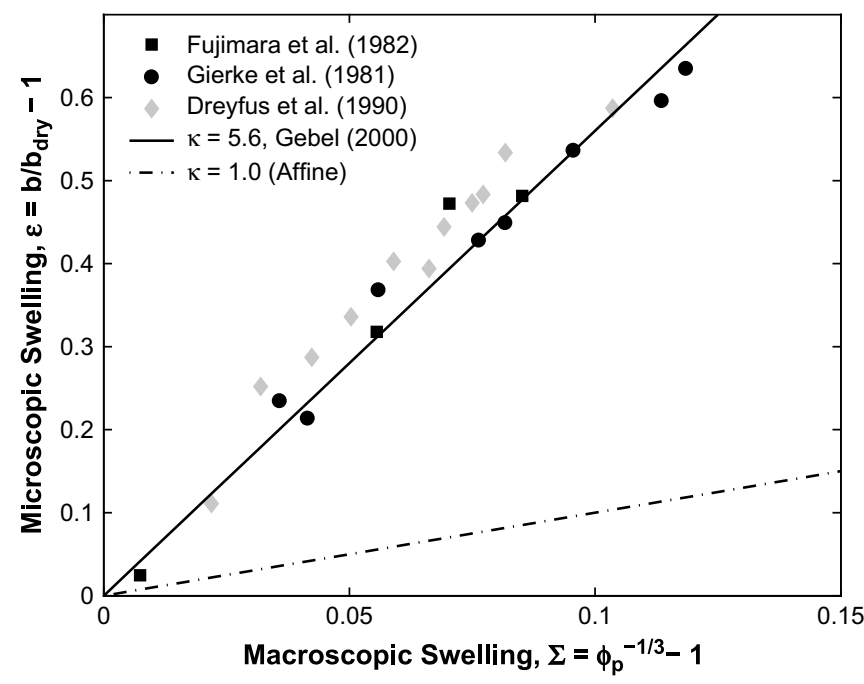

Fig. 2. Microscopic swelling strain, $\varepsilon^{\text {s }}$, given by Eq. (4), as a function of macroscopic swelling strain, $\Sigma^{s}$. The slope of a linear fit through the data is the non-affine swelling ratio, $\kappa$. The experimental data for PFSA membranes of 1100 EW are from Fujimara et al. [20], Dreyfus et al. [22] and Gebel [11] (solid line with slope of $K=5.6$ ) and of $1200 \mathrm{EW}$ are from Gierke et al. [19]. The water content data in the references are converted to the macroscopic swelling strain, $\Sigma^{\mathrm{s}}=\phi_{\mathrm{p}}^{-1 / 3}-1$, assuming isotropic swelling.

a simplified, generalized form: $E_{\mathrm{dry}}=E_{\mathrm{pm}}\left(1-\phi_{\mathrm{c}}^{\mathrm{dry}^{(n-1) / n}}\right)$ where $n=2$ for the cylindrical RVE and $n=3$ for the spherical RVE. This approach to calculating Young's modulus of PFSA membranes is studied in detail in our previous work [63].

\section{Results for swelling pressure}

In generating results, we will focus primarily on PFSA membranes of $\mathrm{EW}=1100$, for which the literature has the most experimental sorption data $[11,18-22,29,33-35]$. In order to quantitatively characterize the non-affine swelling behavior of $1100 \mathrm{EW}$ PFSA membranes, the experimental scattering data from Fujimara et al. [20], Dreyfus et al. [22] and Gebel [11] are used since among the studies in the literature, these are the ones that explicitly report the inter-cluster distance at various degrees of swelling. For comparison purposes, data for 1200 EW PFSA membranes from Gierke et al. [19] are also included and all the water content data are converted to the macroscopic swelling strain, $\Sigma^{\mathrm{s}}=\phi_{\mathrm{p}}^{-1 / 3}-1$ (assumed isotropic). The microscopic swelling strain, $\varepsilon^{\mathrm{s}}$, is calculated from the reported inter-cluster distance (or Bragg distance), which is equivalent to the RVE outer radius in the models (Eq. (4) $)^{1}$ and plotted as a function of macroscopic swelling strain, $\Sigma^{S}$ in Fig. 2. The experimental data from the various researchers all follow a similar linear trend with an average slope of 5.4-5.7. This slope corresponds to the non-affine swelling ratio, $\kappa$ defined in Eq. (6). Thus, in the following calculations we will use $\kappa=5.6$, which is the same non-affine swelling ratio reported previously by Gebel [11]. Similar values can also be derived from more recently published data in the literature. For example, the scattering data for PFSA membranes by Rubatat et al. [33] suggest a value of $\kappa=4.5-6.0$ in the range of water volume fractions considered here.

Assuming the non-affine swelling ratio of 5.6, the stretch in the deformed polymer matrix, $\Lambda_{\mathrm{pm}}$, is plotted as a function of water

\footnotetext{
${ }^{1}$ For the studies in which the inter-cluster distance in the dry state is not specifically given, the distance is determined from extrapolation of the data to zero water volume fraction.
} 


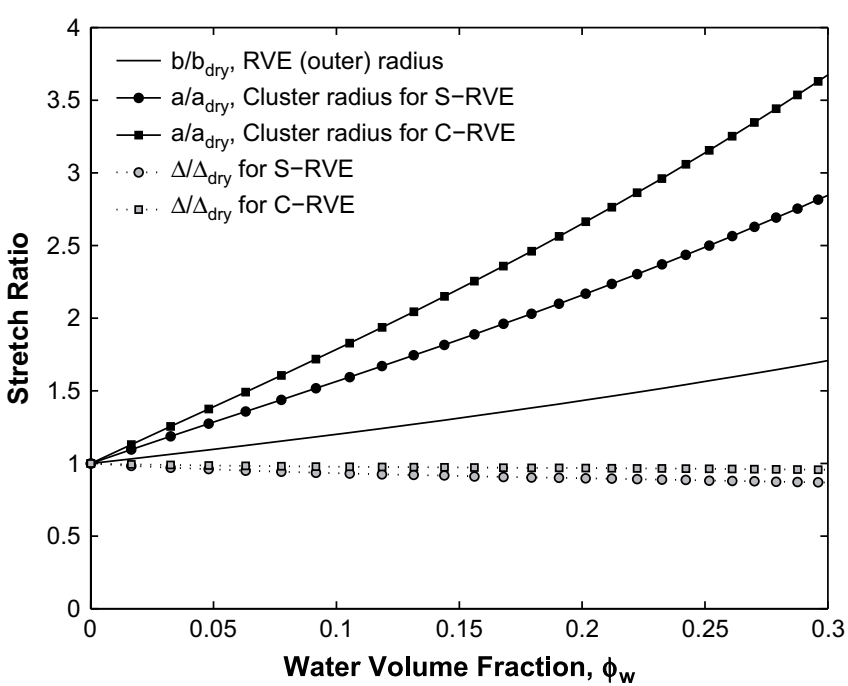

Fig. 3. Change in the outer radius (equal to the half of the inter-cluster distance) and the inner (cluster) radius of the RVEs plotted as a function of water volume fraction. The stretches in the elastic spring representing the compression in the polymer matrix are shown for both spherical RVE (Fig. 1B) and cylindrical RVE (Fig. 1C).

volume fraction for both RVEs in Fig. 3. At the microscopic level, swelling causes compression of the polymer matrix in the radial direction between the growing clusters. This behavior is accounted for in the model as radial springs where the strain in the spring is always negative $\left(\Lambda_{\mathrm{pm}}-1<0\right)$. The change in the RVE radius, $b / b^{\mathrm{dry}}$, and the change in the cluster radius, $a / a^{\mathrm{dry}}$, (which can be calculated using from $b / b^{\text {dry }}$ and Eq. (3)) are also included in the figure for comparison. For non-affine swelling, the change in the length of the spring results from the combination of two mechanisms: (i) the increase in the cluster radius due to the water uptake, which is always related to the RVE radius through the cluster volume fraction; and (ii) the coalescence of the clusters causing an additional increase in the radius of the RVE. For a given water volume fraction, the rate of increase in the cluster radius is always more than that of the RVE radius. This observation is in agreement with the results of the clustering model developed by Dreyfus [67] who showed that the cluster radius increases more rapidly than the inter-cluster distance.

From Eq. (7), we can determine that, $\phi_{c}^{\text {dry }}=+0.0762$ for $\mathrm{EW}=1100$ membrane. Therefore, the polymer matrix modulus in terms of Young's modulus of the dry membrane can be calculated as $E_{\mathrm{pm}}=E_{\mathrm{dry}} /\left(1-\phi_{\mathrm{c}}^{\mathrm{dry}^{(n-1) / n}}\right) \equiv \alpha E_{\mathrm{dry}}$, where the proportionality factor $\alpha$ comes out to be 1.22 for the spherical RVE (S-RVE) $(n=3)$ and 1.38 for the cylindrical RVE (C-RVE) $(n=2)$. Consequently, we can rewrite Eq. (12) in terms of $E_{\text {dry }}$ instead of $E_{\mathrm{pm}}$

$\frac{\Delta P}{E_{\mathrm{dry}}}=\alpha\left\{\left(\left(1+\kappa \phi_{\mathrm{p}}^{-1 / 3}-1\right)\right]\left(\frac{1-\phi_{\mathrm{c}}^{1 / n}}{K_{-}^{\mathrm{dry}}{ }^{1 / n}}\right)(-1\}\right)$

The predictions of Eq. (13) for non-affine swelling $(\kappa=5.6)$ as a function of water volume fraction are depicted as the dashed lines in Fig. 4 for both the S-RVE and C-RVE. Affine swelling ( $\kappa=1$, dotted lines) predicts much higher pressures than non-affine swelling (Fig. 4). We also include the predictions of Eq. (13) for $E_{\mathrm{pm}}=E_{\mathrm{dry}}$, by setting $\alpha=1$ (shown by the solid lines). These results show how the model predictions are affected if Young's modulus of the dry membrane, $E_{\mathrm{dry}}$, is used as the modulus of the elastic springs connecting the clusters rather than the modulus of the hypothetical polymer matrix, $E_{\mathrm{pm}}$. This simplification has a relatively minor effect on the swelling pressure results, showing that the effects of

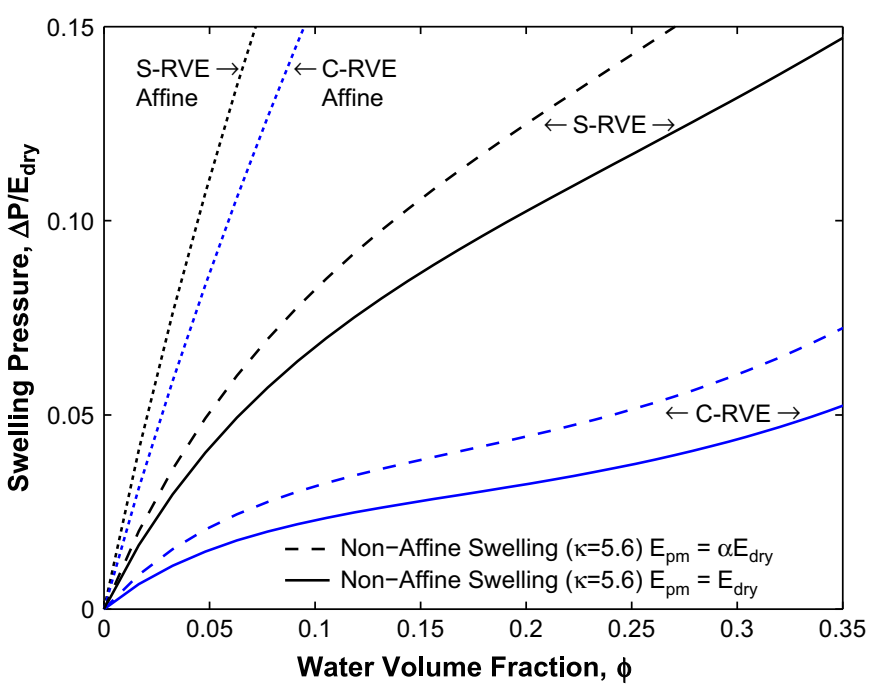

Fig. 4. Predictions of the proposed model for the normalized swelling pressure, $\Delta P /$ $E_{\mathrm{dry}}$, as a function of water volume fraction for affine $(\kappa=1)$ and non-affine swelling $(\kappa=5.6)$ shown for both spherical RVE (S-RVE) and cylindrical RVE (C-RVE). The predictions of the model for the case $\alpha=1$, i.e. Young's modulus of the polymer matrix is equal to that of dry membrane, $E_{\mathrm{pm}}=E_{\mathrm{dry}}$, are also included.

the non-affine swelling ratio and RVE geometry are much more significant than the effect from the modulus correction factor, $\alpha$ (Fig. 4). The shear modulus of the dry membrane, $G_{\mathrm{dry}}$, can also be incorporated into the model, by using the relationship $E_{\mathrm{dry}}=G_{\mathrm{dry}}(1+2 \nu)$ where $\nu$ is Poisson's ratio (can be set to 0.5 ). The relationship between the normalized swelling pressure, $\Delta P / G_{\mathrm{dry}}$, and the water volume fraction is depicted in Fig. 5. For comparison, the prediction of the model developed by Freger [54] from the statistical theory of polymer elasticity is also included. The result of this expression (shown in Eq. (2), [54]) falls between the spherical and cylindrical RVEs in the current formulation.

In this work, we have replaced the polymer matrix material with idealized elastic springs, instead of treating it as a continuum as is done elsewhere in the literature $[18,41,53]$. For example, for the

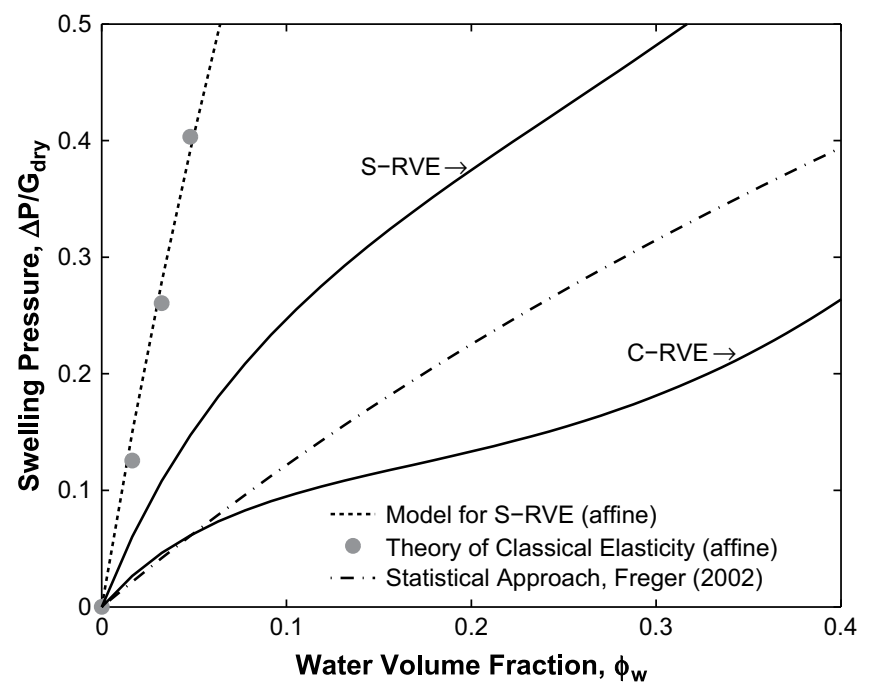

Fig. 5. Predictions of the proposed model for the swelling pressure normalized by shear modulus, $\Delta P / G_{\mathrm{dry}}$, as a function of water volume fraction for the case of nonaffine swelling $(\kappa=5.6)$ of both spherical and cylindrical RVEs. Affine swelling prediction of the proposed model and the results obtained from classical elasticity are shown for the spherical RVE. For comparison, the prediction of Freger's model [54] is also included. 
spherical RVE (Fig. 1B), the pressure field in the solid matrix for an expanding cavity can be determined in spherically symmetric coordinates using the theory of linear elasticity [68]. The problem definition results in an affine solution and the average pressure in the matrix is depicted by the open circles in Fig. 5 . The resulting pressure is similar to the predictions of the proposed model with affine swelling $(\kappa=1)$. Thus, we see that the limitation of the classical elasticity approach comes from the assumption of an RVE with a fixed quantity of polymer, which results in affine swelling and therefore predicts unreasonably high swelling pressures.

In the previous paragraphs, we discussed the relationship between the normalized swelling pressure and the water volume fraction. However, to calculate the actual values of the swelling pressure requires knowledge of Young's modulus of the dry membrane. Using our experimental data [69] for Young's modulus of Nafion ${ }^{\circledR} 112$ membranes ${ }^{2}(1100 \mathrm{EW})$ we showed in our previous work [63] that the following empirical formula can be used to approximate Young's modulus of dry membrane in the temperature range of $25-85^{\circ} \mathrm{C}$ :

$E_{\text {dry }}=1000-2.5 T$

where $T$ is the absolute temperature. Within the framework of this study, Eq. (14) suggests that for a given water content, the swelling pressure will decrease with increasing temperature assuming the parameters $\alpha$ and $\kappa$ are temperature independent.

In the literature, the water content in the membrane is usually represented by $\lambda$, the number of water molecules per $\mathrm{SO}_{3}^{-}$group. For a given ionomer, the relationship between the water volume fraction, $\phi_{\mathrm{w}}$, and the water content, $\lambda$, is given by

$\phi_{\mathrm{w}}=\frac{\lambda}{\bar{V}_{\mathrm{p}} / \bar{V}_{\mathrm{w}}+\lambda}$.

where $\bar{V}_{\mathrm{w}}$ and $\bar{V}_{\mathrm{p}}$ are the molar volume of water (taken to be $\bar{V}_{\mathrm{w}}=18 \mathrm{~cm}^{3} / \mathrm{mol}$ ) and the polymer ionomer (see Eq. (7)). The water uptake behavior of PFSA membranes is typically characterized by sorption isotherms which define the relationship between the water content, $\lambda$, and the vapor activity of water in the surrounding air, $a_{\mathrm{w}}$, (or relative humidity, $\mathrm{RH}$ ) at a given temperature $[5,24,40,42,44,45,70,71]$.

In equilibrium, the confining pressure applied by the polymer matrix is balanced by osmotic swelling pressure, or the tendency of external moisture to diffuse into the membrane and increase the swelling. Therefore, once the relationship between the swelling pressure and the water volume fraction is established, this relationship can be used in thermodynamic phase equilibrium equations to determine the sorption isotherms. Thus, in order to further analyze the proposed swelling pressure formulation and to understand the effects of the proposed RVEs and temperature dependence on water uptake, we will compare our model predictions to the sorption behavior of PFSA membranes based on the theoretical, thermodynamic approaches used and discussed elsewhere in the literature [40-42].

\section{Investigation of sorption behavior}

\subsection{Thermodynamic modeling of sorption isotherms}

For PFSA polymer and water in equilibrium, the difference between the logarithms of the activity of the water external to the

\footnotetext{
2 Nafion $^{\circledR}$ is a registered trademark of E.I. DuPont De Nemours \& Co. Nafion ${ }^{\circledR}$ membrane is a commercially available PFSA-based membrane commonly used in fuel cell applications.
}

polymer, $a_{\mathrm{w}}$, and the activity internal to the polymer, $a_{\mathrm{p}}$, is proportional to the product of the osmotic pressure, $\Pi$, and the molar volume of water, $\bar{V}_{\mathrm{w}}[41,72]$

$R T \ln a_{\mathrm{w}}=R T \ln a_{\mathrm{p}}+\Pi \bar{V}_{\mathrm{w}}$,

where $T$ is the absolute temperature and $R$ is the universal gas constant. Thus, the phase equilibrium between the PFSA polymer membrane and the water activity can be described by

$\ln \frac{a_{\mathrm{p}}}{a_{\mathrm{w}}}=-\frac{\bar{V}_{\mathrm{w}}}{R T} \Pi$

The water content within the membrane consists of chemically bound water, $\lambda^{\mathrm{B}}$, and free water, $\lambda^{\mathrm{F}}[24,40-42,51]$. The water activity within the membrane is approximately equal to the mole fraction of the free water within the membrane, i.e. $a_{\mathrm{p}}=\lambda^{\mathrm{F}}\left(1+\lambda^{\mathrm{F}}\right)[40-42]$.

At equilibrium, the osmotic pressure is equal to the change in the compressive pressure, $\Delta P$, given in Eq. (13). Thus, the pressure formulation proposed in the previous section can be used in Eq. (17) by setting $\Pi=\Delta P$. Consequently, the following implicit expression (from Eq. (17)) can be used to analyze the sorption behavior of PFSA membrane

$\frac{\lambda^{\mathrm{F}}}{1+\lambda^{\mathrm{F}}}=\frac{\lambda-\lambda^{\mathrm{B}}}{1+\lambda-\lambda^{\mathrm{B}}}=a_{\mathrm{w}} \exp \left[-\frac{\bar{V}_{\mathrm{w}}}{R T} \Delta P\left(\phi_{\mathrm{w}}(\lambda), T\right)\right]$.

The bound water as a function of water activity can be approximated using the expression given in the work of Choi et al. [40,42] on their physicochemical treatise of the solvation of ionic groups in PFSA membranes:

$\left.\lambda^{\mathrm{B}}\left(a_{\mathrm{w}}\right)=1.8 \frac{K_{1} a_{\mathrm{w}}}{1-a_{\mathrm{w}}} \frac{1-(\nu+1) a_{\mathrm{w}}^{\nu}+\nu a_{\mathrm{w}}^{\nu+1}}{1+\left(K_{1}-1\right) a_{\mathrm{w}}-K_{1} a_{\mathrm{w}}^{\nu+1}}\right)$.

where the model constants are $K_{1}=100$ and $\nu=4-5$ for PFSA membranes with $1100 \mathrm{EW}$. For the swelling pressure term in Eq. (18) the water volume fraction, $\phi_{\mathrm{w}}$, is converted to total water content, $\lambda$, not the free water $\lambda^{\mathrm{F}}$ : even though some water molecules are thermodynamically bound, the entire swollen-ionic domains are treated as zero-stiffness domains within the context of our mechanics-based pressure modeling [63].

The Flory-Huggins theory for polymer solutions [55] can also be used to describe the swelling equilibrium in PFSA membranes as discussed elsewhere [42,71,73]. This model, however, has an additional parameter, the so-called Flory-Huggins interaction parameter, $\chi$, which characterizes the enthalpic energy of mixing between the polymer and solvent. This parameter has to be determined empirically. For PFSA membranes, the value for $\chi$ has been determined to be $1.0-1.5$ in the literature [42,71]. If we use the Flory-Huggins model with our pressure formulation, the resulting sorption isotherms (not shown) turn out to be very similar to those predicted by Eq. (18) (e.g. Fig. 6) if the interaction parameter is set to $\chi=1.0-1.3$ at $25^{\circ} \mathrm{C}$, which tends to further validate our model. However, we will leave the full investigation of these relationships for future work due to the temperature-dependent nature of $\chi$ and the lack of sorption data at various temperatures.

\subsection{Effect of RVE geometry}

We have solved Eq. (18) for PFSA membranes of EW $=1100$ at $25^{\circ} \mathrm{C}$ using the proposed mechanics-based swelling pressure model for both the spherical and cylindrical RVEs. The resulting sorption isotherms are depicted in Fig. 6 along with experimental data for the water uptake of EW 1100 membranes from Zawodzinski et al. [8,38], Morris and Sun [10], Choi et al. [42], and the 


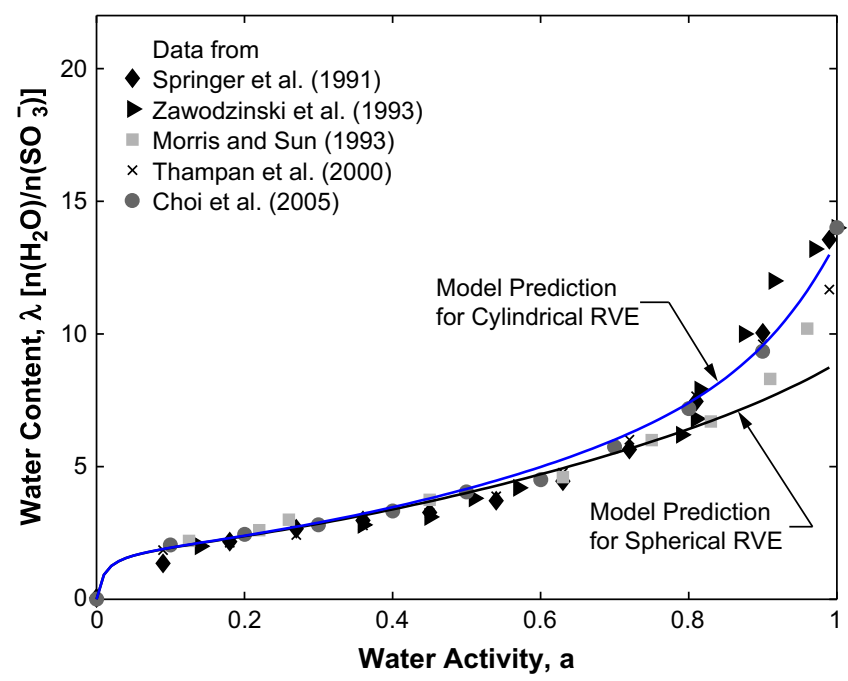

Fig. 6. Sorption isotherms for PFSA membranes of $1100 \mathrm{EW}$ from water vapor at $25{ }^{\circ} \mathrm{C}$ obtained from the predictions of the swelling pressure model for spherical RVE and cylindrical RVE compared with the experimental data from Zawodzinski et al. $[8,38]$, Morris and Sun [10], Choi et al. [42] and the empirical models from Springer et al. [6] and Thampan et al. [44].

empirical models of Springer et al. [6] and Thampan et al. [44]. For water activities below 0.50 , the effect of the RVE geometry on the water uptake behavior is negligible within the scale of the figure. However, at higher water activities, the spherical and the cylindrical RVEs diverge, and the experimental data seem to shift from the S-RVE predictions at intermediate activity $\left(0.5<a_{\mathrm{w}}<0.8\right)$ to the C-RVE predictions at higher activities $\left(a_{\mathrm{w}}>0.8\right)$. A similar shift has been observed in experimental sorption data of PFSA membranes at around $a_{\mathrm{w}}=0.7-0.8$ [8]. They attribute the region with lower slope with the solvation of $\mathrm{SO}_{3}^{-}$ions with water, and the region with higher slope to the swelling of the membrane with additional free water [8]. Our results are also consistent with the findings of Laporta et al. [24], who suggested that that the nanostructure of the water phase in a swollen PFSA might be intermediate between the two limiting shapes of a sphere and cylinder.

\subsection{Effect of temperature}

The effect of temperature on water uptake can be investigated in our model by inputting the empirical relationship between Young's modulus and temperature, Eq. (14), into the swelling pressure formulation, Eq. (13) and using the resulting expression for the pressure in Eq. (18). We will here focus on sorption from saturated water vapor $\left(a_{\mathrm{w}}=1\right)$. However, it has been reported that $[8,46,50]$ the water content in predried ${ }^{3}$ membranes is similar, whether the membrane is equilibrated in saturated water vapor or liquid water. Thus, we will here adopt the results from a number of experimental water uptake studies for predried PFSA membranes equilibrated in liquid water and/or water vapor from Zawodzinski et al. [8,38], Hinatsu et al. [45], Onishi et al. [46], and Thompson et al. [74]. According to these studies, the water content of the predried membrane saturated with water is in the range of $\lambda=12-20$, in close agreement with the predictions of our cylindrical RVE model (Fig. 7). The results of our calculation are plotted in Fig. 7, which shows that water uptake of a predried membrane saturated with

\footnotetext{
${ }^{3}$ Predried here refers to membranes dried at $80-105^{\circ} \mathrm{C}$ for $1-24 \mathrm{~h}$ (sometimes in vacuum) after the standard pretreatment procedure. For details, interested reader is urged to see the original publications.
}

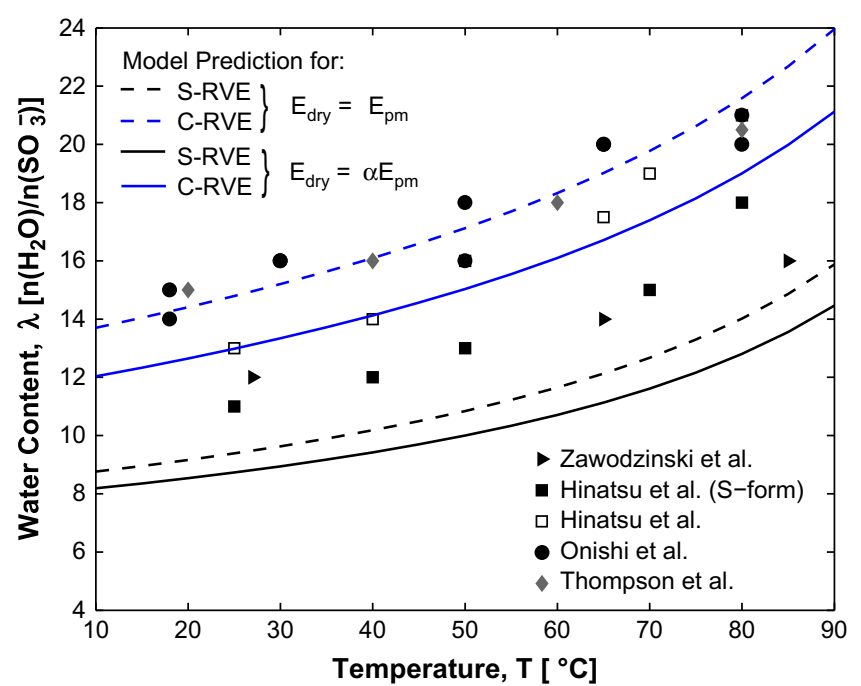

Fig. 7. Water content predictions of the proposed model for a saturated membrane $(a=1)$ are plotted as a function of temperature using spherical RVE and cylindrical RVE compared with the water uptake data from literature; Zawodzinski et al. $[8,38]$, Hinatsu et al. [45] and Onishi et al. [46] for predried PFSA membranes of $1100 \mathrm{EW}$ in equilibrium with saturated water vapor or liquid water. All the data shown here are for the membranes predried at $105{ }^{\circ} \mathrm{C}$ except the S-form membrane of Hinatsu et al. [45] which is predried at $80^{\circ} \mathrm{C}$.

water $\left(a_{\mathrm{w}}=1\right)$ increases with increasing temperature for both the C-RVE and S-RVE. This prediction is consistent with the experimental observations.

Onishi et al. [46] explained this temperature-water content relationship, by noting that the predried membrane is initially free of water, i.e. both bound and free water are absent, and therefore somehow partially constrained, limiting its ability to uptake water. They further explain that this apparent constraint relaxes with increasing temperature and water content. Similarly, in a study by Satterfield and Benziger [75] on the water sorption dynamics of predried PFSA membranes, the rate of water sorption was found to increase with increasing temperature, which they attributed to the relaxation of the polymer matrix. Therefore, there is experimental evidence to suggest that the water uptake is influenced by the temperature effect on the behavior of the polymer matrix.

In this work, we have demonstrated a similar effect using our mechanics-based model. According to our model, the increase in water uptake with increasing temperature is due to the decrease in Young's modulus of the polymer matrix in the membrane. Thus, our model predictions for idealized membrane coincide with the sorption behavior seen in predried membranes, even though pretreatment was not explicitly taken into account. Nevertheless, in the model formulation, we assumed that the swelling pressure is initially zero and that the clusters are completely dry before water uptake begins. Similarly, the $\kappa$ value of 5.6, was determined based on the initial inter-cluster distance in a dry membrane, not in a membrane at ambient conditions [11,19,20,22,33]. Consequently, our model was developed from a set of assumptions consistent with a membrane in the predried state.

\subsection{Effect of initial (residual) water in the membrane}

As discussed in the previous section, the maximum water content in previously predried membranes is similar whether the membrane is equilibrated in water vapor or liquid water $[8,46,50,74]$. In contrast to this, for membranes that are not predried (at elevated temperatures for a certain period of time), but subjected to standard treatment procedure, the water content is 
$\lambda=21-24$ in liquid water $[10,38,45,46]$. This discrepancy has been attributed to the thermal history of the polymer, which has been shown to play a key role in the water uptake capability of PFSA membranes $[8,46]$. Similar observations were actually made earlier by Zawodzinski et al. [8], who suggest that predrying the membranes leads to the breakup of the ionic clusters, whereas in non-predried membranes, hydrated ionic clusters are present initially. Therefore, the existence of any residual water in the (nonpredried) membrane influences the subsequent water uptake behavior.

Because different researchers use different membrane pretreatment procedures and several different experimental measurement techniques, it is difficult to compare the available sorption data in the literature, as pointed out by Onishi et al. [46]. Experimental data from Roche et al. [49], Laporta et al. [24] and Kim et al. [34] for the sorption of non-predried PFSA from water vapor are plotted in Fig. 8. It follows from the figure that water uptake for non-predried membranes is higher than for predried membrane (Fig. 6) for a given water activity.

In order to further investigate this phenomenon, we extend our swelling pressure formulation for the case of non-predried membrane by assuming that there exists a small amount of residual (e.g. bound) water in the membrane initially. This means that, initially, the cluster volume fraction is the sum of the volume fraction of the $\mathrm{SO}_{3}^{-}$groups, $\phi_{\mathrm{c}}^{\mathrm{dry}}$, and the initial water volume fraction, $\phi_{\mathrm{w}}^{0}$, i.e. $\phi_{\mathrm{c}}^{0}=\phi_{\mathrm{c}}^{\mathrm{dry}}+\phi_{\mathrm{w}}^{0}$. As a result, the initial length of the "springs" changes and, according to Eq. (10), a different stretch will be generated upon swelling. When water is already present in the clusters, less additional pressure is generated in the polymer matrix for a given amount of additional water. Following this idea, the swelling pressure from Eq. (12) is recalculated (assuming all other parameters are the same) and new sorption isotherms are generated (Fig. 8). The initial water volume fractions of (i) $\phi_{\mathrm{w}}^{0}=0.1$, and (ii) $\phi_{\mathrm{w}}^{0}=0.125$ are used, corresponding to water content of $\lambda \approx 1$ and $\lambda=1.5-2.5$, respectively. As seen from the figure, the proposed model can capture the increased water uptake for the membranes with initial water content. Therefore, our mechanics-based modeling approach can account for the effect of residual water in the membrane on the subsequent sorption behavior.

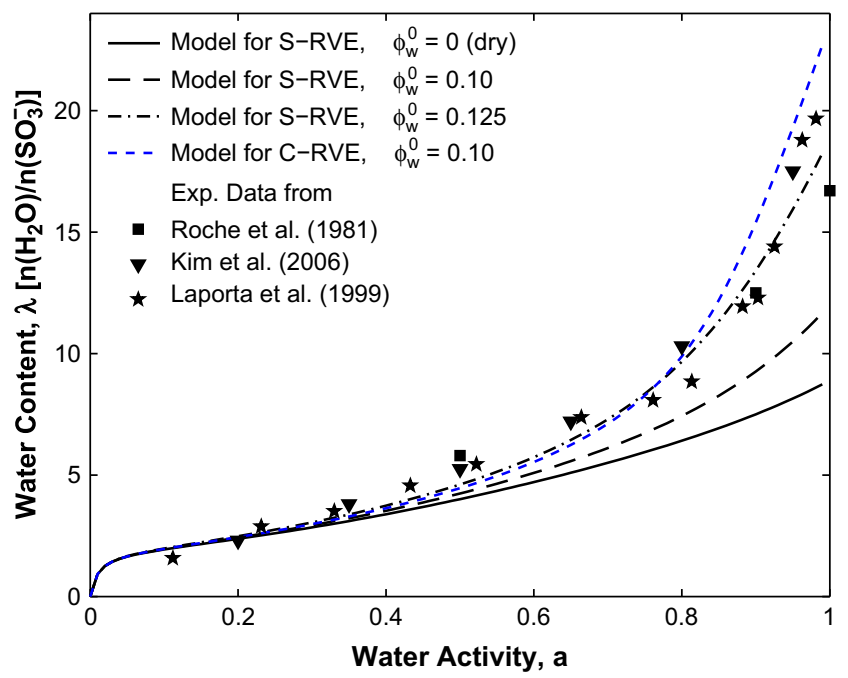

Fig. 8. Predictions of the proposed model for the sorption isotherms of non-predried PFSA membrane at room temperature with initial water volume fraction of $\phi_{w}^{0}=0.10$ and 0.125 for spherical RVE (S-RVE) and $\phi_{\mathrm{w}}^{0}=0.10$ for cylindrical RVE (C-RVE). Results for predried membrane $\left(\phi_{\mathrm{w}}^{0}=0\right)$ are also shown for S-RVE. For comparison, measured sorption data for non-predried PFSA membranes are included from Roche et al. [49], Laporta et al. [24] and Kim et al [34].

\subsection{Effect of Young's modulus of dry membrane}

The effect of the membrane Young's modulus in the dry state, $E_{\text {dry, }}$ on its maximum water uptake capacity under saturated conditions (at $T=25^{\circ} \mathrm{C}$ ) is depicted in Fig. 9. For a stiffer polymer backbone, the elastic counter pressure exerted on the water domains increases, resulting in less water uptake. The predicted water content, $\lambda$, also depends on the assumed RVE; cylindrical water domains absorb more water than the spherical ones. The effect of the relationship between the polymer backbone modulus $\left(E_{\mathrm{pm}}\right)$ and the dry membrane's modulus $\left(E_{\mathrm{dry}}\right)$ is also depicted in the figure. When we assume that $E_{\mathrm{pm}}=E_{\mathrm{dry}}$, the model predicts more water absorption than when we assume $E_{\mathrm{pm}}=\alpha E_{\mathrm{dry}},(\alpha=1.22$ for $\mathrm{S}$ RVE and 1.38 for C-RVE) since in the latter case, the backbone does not contain the $\mathrm{SO}_{3}^{-}$ionic groups, and therefore has a higher overall modulus.

\section{Discussion}

Within the framework of this study, the increase in the cluster radius is due to the simultaneous increase in water content, $\lambda$, and increase in the number of $\mathrm{SO}_{3}^{-}$groups per cluster, $\mathrm{N}_{\mathrm{SO}_{3}}$. However, the increase in $N_{\mathrm{SO}_{3}}$, due to cluster coalescence (non-affine swelling) has the effect of reducing the swelling pressure relative to affine behavior. This phenomenon can be attributed to the releasing of constraints in the polymer matrix, similar to the arguments by Onishi [46] cited in Section 5.5. Also, $N_{\mathrm{SO}_{3}}$ has been measured to be 26-30 for a dry (predried) Nafion 1100 membrane and 76-90 for a swollen membrane at water volume fraction $\phi_{\mathrm{w}} \approx 0.3[18,19,21-$ 23] corresponding to a value of the ratio $N_{\mathrm{SO}_{3}} / N_{\mathrm{SO}_{3}}^{\mathrm{dry}}=2.5-3.5$. The current model predicts that $N_{\mathrm{SO}_{3}} / N_{\mathrm{SO}_{3}}^{\mathrm{dry}}=3.5$ for $\phi_{\mathrm{W}}=0.3$ (Eq. (9) with $\kappa=5.6$ ) surprisingly consistent with the literature values.

A reduction in the non-affine swelling parameter, $\kappa$, might correspond to an increase in mechanical constraint. As a limiting case, affine swelling $(\kappa=1)$ may be attributed to the sorption of a fully constrained membrane for which model predicts $\lambda=5-7$. This value is slightly higher than the amount of bound water, and much lower than that for unconstrained membrane $(\lambda=13-15)$ exhibiting similar trends with other model predictions $[70,76]$. However, in order to fully understand the physical implications of

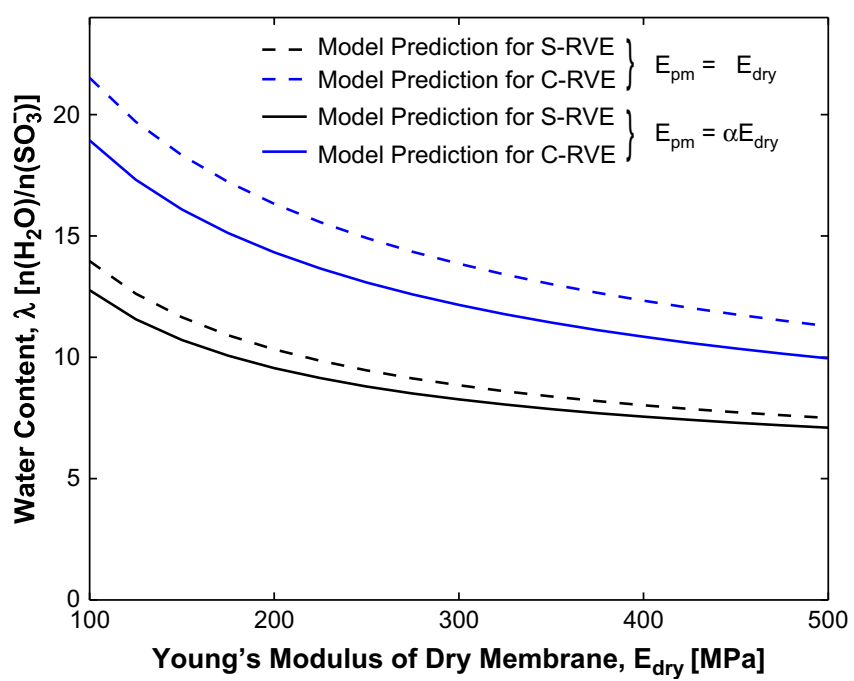

Fig. 9. Model predictions for the maximum water uptake capacity of the PFSA membrane as a function of membrane's Young's modulus in dry state shown for the spherical (S-RVE) and the cylindrical (C-RVE) geometries for (i) $E_{\mathrm{dry}}=E_{\mathrm{pm}}$ (solid lines) and (ii) $E_{\mathrm{dry}}<E_{\mathrm{pm}}$ (dashed lines). 
changing $\kappa$, the relationship between water uptake and the nanostructure of a constrained membrane (e.g. cluster size, inter-cluster distance) must be investigated more fully.

In previous studies, the radius of the clusters has been shown to increase from $\sim 1 \mathrm{~nm}$ in dry membrane up to 4-6 $\mathrm{nm}$ depending on the water content $[11,18,19,21-23]$. Since normalized dimensions (e.g. stretch, strain) are used in the model, the pressure formulation is independent of the actual values of the cluster radii. Therefore, with a little modification, the proposed model can possibly be used to investigate the effects of a non-uniform cluster size distribution (CSD) and water content gradients [20,31,77]. Also, the modeling framework presented here may prove to be helpful for studying the time-dependent swelling behavior, which is important to the understanding of sorption dynamics.

We see that this mechanics-based model is able to capture and explain a number of phenomena associated with swelling of PFSA from a mechanical perspective. The model is based on the following nano-structural and material properties: (i) the assumed RVE geometry, (ii) the non-affine swelling behavior and (iii) Young's modulus and molecular weight of the polymer. Consequently, we believe that the proposed modeling approach provides a flexible framework that can be used to investigate the swelling pressure and sorption behavior of other ionomers with various nanostructures and non-affine swelling behaviors.

\section{Conclusion}

We developed an analytical mechanics-based modeling framework using the concept of non-affine swelling as applied to several, simple RVEs to describe the swelling pressure of PFSA ionomers. This work is aimed at explaining and correlating experimental observations of non-affine swelling with the nanostructure and temperature-dependent sorption behavior using an analytical model based on a mechanical (rather than energetic) approach. Thus, the water uptake behavior is discussed to demonstrate further capabilities of the proposed formulation and to examine other effects of the model parameters and RVE geometry. In this work, we focus primarily on PFSA membrane to validate our model due to the large body of experimental evidence on both the nanostructure and sorption behavior of this ionomer. However, the modeling framework can easily be extended to characterize other solvent-polymer systems and ionomers provided that the basic physical and structural properties are known.

The deformation of the polymer matrix due to swelling is modeled by hypothetical, linear elastic spring elements located between the inner and outer radii of the RVEs. Thus, the swelling pressure is assumed to be proportional to the spring stiffness, (the temperature-dependent Young's modulus of the polymer matrix) which can account for the temperature-dependent sorption behavior. According to the proposed model, swelling pressure increases with increasing water content due to the growth of the clusters, which in turn, causes compression in the polymer matrix. We have shown that the non-affine swelling behavior reduces the matrix pressure relative to a purely elastic affine deformation. Therefore, the affine swelling assumption (as in the classical elasticity formulation) significantly over-predicts the swelling pressure developed in PFSA membranes.

At lower water content, both RVEs examined in this work generate theoretical sorption isotherms, which accurately predict the experimental data. However, the cylindrical RVE appears to give better predictions at higher water contents. Moreover, as the temperature increases, the model predicts an increase in water uptake, in agreement with the experimental observations for the sorption behavior of predried PFSA membranes found in the literature. Also, residual water present in a non-predried membrane initially, increases the subsequent water uptake. The water uptake is found to decrease with increasing backbone stiffness and with decreasing degree of non-affine swelling. These two effects are important since they indicate that the water uptake behavior, which is vital, for example in fuel cell applications, can be influenced by controlling the mechanical properties of the membrane backbone, or altering the mechanical constraints on the membrane swelling.

\section{Acknowledgements}

This research is part of the CIRRUS project supported by the US Department of Energy Hydrogen Program.

\section{References}

[1] Mathias MF, Makharia R, Gasteiger HA, Conley JJ, Fuller TJ, Gittleman CJ, et al. The Electrochemical Society Interface 2005;14(3):24-35.

[2] Macknight WJ, Earnest TR. Macromolecular Reviews Part D: Journal of Polymer Science 1981;16:41-122.

[3] Pivovar BS. Polymer 2006;47(11):4194-202.

[4] Rajendran RG. MRS Bulletin 2005;30(8):587-90.

[5] Mauritz KA, Moore RB. Chemical Reviews 2004;104(10):4535-85.

[6] Springer TE, Zawodzinski TA, Gottesfeld S. Journal of the Electrochemical Society $1991 ; 138(8): 2334-42$.

[7] Weber AZ, Newman J. Journal of the Electrochemical Society 2004;151(2): 311-25.

[8] Zawodzinski TA, Derouin C, Radzinski S, Sherman RJ, Smith VT, Springer TE, et al. Journal of the Electrochemical Society 1993;140(4):1041-7.

[9] Hsu WY, Gierke TD. Journal of Membrane Science 1983;13(3):307-26.

[10] Morris DR, Sun XD. Journal of Applied Polymer Science 1993;50(8):1445-52.

[11] Gebel G. Polymer 2000;41(15):5829-38.

[12] Fontanella JJ, Edmondson CA, Wintersgill MC, Wu Y, Greenbaum SG. Macromolecules 1996;29(14):4944-51.

[13] Eikerling M, Kornyshev AA, Kucernak AR. Physics Today 2006;59(10):38-44.

[14] Tang Y, Kusoglu A, Karlsson AM, Santare MH, Cleghorn S, Johnson WB. Journal of Power Sources 2008;175(2):817-25.

[15] Kusoglu A, Karlsson AM, Santare MH, Cleghorn S, Johnson WB. Journal of Power Sources 2007;170(2):345-58.

[16] Kusoglu A, Karlsson AM, Santare MH, Cleghorn S, Johnson WB. Journal of Power Sources 2006;161(2):987-96.

[17] Gebel G, Diat O. Fuel Cells 2005;5(2):261-76.

[18] Hsu WY, Gierke TD. Macromolecules 1982;15(1):101-5.

[19] Gierke TD, Munn GE, Wilson FC. Journal of Polymer Science, Polymer Physics Edition 1981;19(11):1687-704.

[20] Fujimura M, Hashimoto T, Kawai H. Macromolecules 1982;15(1):136-44.

[21] Gebel G, Lambard J. Macromolecules 1997;30(25):7914-20.

[22] Dreyfus B, Gebel G, Aldebert P, Pineri M, Escoubes M, Thomas M. Journal de Physique 1990;51(12):1341-54.

[23] Orfino FP, Holdcroft S. Journal of New Materials for Electrochemical Systems 2000;3(4):285-90.

[24] Laporta M, Pegoraro M, Zanderighi L. Physical Chemistry Chemical Physics 1999;1(19):4619-28

[25] Roche EJ, Pineri M, Duplessix R. Journal of Polymer Science, Part B: Polymer Physics 1982;20(1):107-16.

[26] Eisenberg A. Macromolecules 1970;3(2):147-54.

[27] Dreyfus B. Journal of Polymer Science, Part B: Polymer Physics 1983;21(11): 2337-47.

[28] Schmidt-Rohr K, Chen Q. Nature Materials 2008;7(1):75-83.

[29] Termonia Y. Polymer 2007;48(5):1435-40.

[30] Eikerling M, Kornyshev AA, Stimming U. Journal of Physical Chemistry B 1997;101(50):10807-20.

[31] Vishnyakov A, Neimark AV. Journal of Physical Chemistry B 2001; 105(39):9586-94.

[32] Hsu WY, Gierke TD, Molnar CJ. Macromolecules 1983;16(12):1945-7.

[33] Rubatat L, Rollet AL, Gebel G, Diat O. Macromolecules 2002;35(10):4050-5

[34] Kim MH, Glinka CJ, Grot SA, Grot WG. Macromolecules 2006;39(14):4775-87.

[35] James PJ, Elliott JA, McMaster TJ, Newton JM, Elliott AMS, Hanna S, et al Journal of Materials Science 2000;35(20):5111-9.

[36] Gruger A, Regis A, Schmatko T, Colomban P. Vibrational Spectroscopy 2001; 26(2):215-25.

[37] Haubold HG, Vad T, Jungbluth H, Hiller P. Electrochimica Acta 2001;46(10-11): 1559-63.

[38] Zawodzinski TA, Springer TE, Uribe F, Gottesfeld S. Solid State Ionics 1993; 60(1-3):199-211.

[39] Weber AZ, Newman J. Journal of the Electrochemical Society 2003;150(7):1008-15.

[40] Choi PH, Datta R. Journal of the Electrochemical Society 2003;150(12):E601-7.

[41] Mauritz KA, Rogers CE. Macromolecules 1985;18(3):483-91.

[42] Choi P, Jalani NH, Datta R. Journal of the Electrochemical Society 2005;152(3): E84-9. 
[43] Gusler GM, Cohen Y. Industrial and Engineering Chemistry Research 1994;33(10):2345-57.

[44] Thampan T, Malhotra S, Tang H, Datta R. Journal of the Electrochemical Society 2000; $147(9): 3242-50$

[45] Hinatsu JT, Mizuhata M, Takenaka H. Journal of the Electrochemical Society 1994;141(6):1493-8.

[46] Onishi LM, Prausnitz JM, Newman J. Journal of Physical Chemistry B 2007;111(34):10166-73.

[47] Satterfield MB, Majsztrik PW, Ota H, Benziger JB, Bocarsly AB. Journal of Polymer Science, Part B: Polymer Physics 2006;44(16):2327-45.

[48] Gebel G, Aldebert P, Pineri M. Polymer 1993;34(2):333-9.

[49] Roche EJ, Pineri M, Duplessix R, Levelut AM. Journal of Polymer Science, Part B: Polymer Physics 1981;19(1):1-11.

[50] Siu A, Schmeisser J, Holdcroft S. Journal of Physical Chemistry B 2006;110(12):6072-80.

[51] Thompson EL, Capehart TW, Fuller TJ, Jorne J. Journal of the Electrochemical Society 2006;153(12):A2351-62.

[52] Aldebert P, Dreyfus B, Pineri M. Macromolecules 1986;19(10):2651-3.

[53] Zhao XH, Hong W, Suo ZG. Applied Physics Letters 2008;92(5).

[54] Freger V. Polymer 2002;43(1):71-6.

[55] Flory PJ. Principles of polymer chemistry. Ithaca: Cornell University Press; 1953.

[56] Rubinstein M, Colby RH, Dobrynin AV, Joanny JF. Macromolecules 1996;29(1): 398-406.

[57] Nandan D, Venkataramani B, Gupta AR. Langmuir 1993;9(7):1786-93.

[58] Zhao YQ Eichinger BE. Macromolecules 1992;25(25):6996-7002.

[59] Nandan D, Gupta AR. Journal of Physical Chemistry 1977;81(12):1174-9.

[60] Tiihonen J, Laatikainen M, Markkanen I, Paatero E. Industrial and Engineering Chemistry Research 1999;38(12):4832-42.
[61] Gottlieb M, Gaylord RJ. Macromolecules 1984;17(10):2024-30.

[62] Hashin Z. International Journal of Solids and Structures 1985;21(7):711-20.

[63] Kusoglu A, Santare MH, Karlsson AM, Cleghorn S, Johnson WB. Journal of Polymer Science Part B: Polymer Physics 2008;46(22):2404-17.

[64] James HM, Guth E. Journal of Chemical Physics 1943;11(10):455-81.

[65] Treloar LRG. The physics of rubber elasticity. 3rd ed. New York: Oxford University Press; 2005.

[66] Arruda EM, Boyce MC. Journal of the Mechanics and Physics of Solids 1993;41(2):389-412.

[67] Dreyfus B. Macromolecules 1985;18(2):284-92.

[68] Boresi AP, Chong KP. Elasticity in engineering mechanics. 2nd ed. New York: John Wiley \& Sons Inc.; 2000.

[69] Tang Y, Karlsson AM, Santare MH, Gilbert M, Cleghorn S, Johnson WB. Materials Science and Engineering A 2006;425(1-2):297-304.

[70] Weber AZ, Newman J. AIChE Journal 2004;50(12):3215-26.

[71] Futerko P, Hsing IM. Journal of the Electrochemical Society 1999;146(6):2049-53.

[72] Hiemenz PC, Lodge TP. Polymer chemistry. 2nd ed. Boca Raton: CRC Press; 2007.

[73] Takata H, Mizuno N, Nishikawa M, Fukada S, Yoshitake M. International Journal of Hydrogen Energy 2007;32(3):371-9.

[74] Thompson EL, Jorne J, Gu WB, Gasteiger HA. Journal of the Electrochemical Society 2008;155(6):B625-34.

[75] Satterfield MB, Benziger JB. Journal of Physical Chemistry B 2008;112(12): 3693-704.

[76] Nazarov I, Promislow K. Journal of the Electrochemical Society 2007;154(7): 623-30.

[77] Iijima M, Sasaki Y, Osada T, Miyamoto K, Nagai M. International Journal of Thermophysics 2006;27(6):1792-802. 